\title{
Boosting invisible Higgs boson searches by tagging a gluon jet for the gluon fusion process
}

\author{
Won Sang Cho®, ${ }^{*}$ Hyung Do Kim $\odot{ }^{\dagger}$ and Dongsub Lee $\odot^{\ddagger}$ \\ Center for Theoretical Physics, Department of Physics and Astronomy, \\ Seoul National University, Seoul 08826, Korea
}

(Received 17 March 2020; revised 30 July 2020; accepted 12 October 2020; published 3 December 2020)

\begin{abstract}
We propose a novel method in that quark-gluon tagging of the jets emitted as initial state radiation (ISR) can boost searches of invisible Higgs bosons from gluon fusion processes against irreducible electroweak vector boson productions. While quark ISR typically takes up a dominant portion than gluon in the background processes mainly by frequent quark-gluon-initiated hard scatterings at the LHC, the gluon ISR portion in the gluon fusion can be significantly larger in the central region of the detector. Focusing on invisible Higgs boson searches using jet substructure variables capturing the new features, we demonstrate that the Higgs boson from gluon fusion constrains invisible Higgs boson decays the most, over vector boson fusion traditionally known as the most constraining, and the limit on the branching ratio is significantly improved. We summarize with emphasizing that our method has wider implications in the search for new resonances from gluon fusion processes.
\end{abstract}

DOI: 10.1103/PhysRevD.102.115007

\section{INTRODUCTION}

The Higgs boson discovery at the LHC in 2012 completed the Standard Model (SM) as a description of nature in terms of elementary particles and their interactions [1,2], and the precision measurement of the SM Higgs boson couplings is one of the most important tasks for probing new physics and the dynamics of electroweak symmetry breaking of the Universe at future collider experiments [3]. However, Higgs boson precision measurements are highly nontrivial tasks in the existence of huge irreducible backgrounds. In particular, production of electroweak vector bosons (EWVBs), such as $W, Z$, and $\gamma$, comprise a large portion of the irreducible background. This is because (i) massive gauge bosons, $Z$ and $W$, are in mass scales similar with the Higgs boson, (ii) decayed particle contents are the same (or easy to be misidentified) with the Higgs boson decays $(H \rightarrow f \bar{f})$, and (iii) the Higgs boson also decays to a pair of EWVBs with $\operatorname{BR}(H \rightarrow V V) \sim 23 \%$.

Among various production mechanisms of the Higgs boson at the LHC, Higgs boson production via gluon fusion (ggH) $[4,5]$ has the most dominant contribution $(90 \%)$ to

\footnotetext{
wscho@snu.ac.kr

†hdkim@phya.snu.ac.kr

*dongsub93@snu.ac.kr
}

Published by the American Physical Society under the terms of the Creative Commons Attribution 4.0 International license. Further distribution of this work must maintain attribution to the author(s) and the published article's title, journal citation, and DOI. Funded by SCOAP. the total production cross sections. The ggH process is a very unique process, in that it can transform between the state of QCD force carriers and electroweak bosons via quark loops, and not leaving any other QCD remnants at leading order (LO), so its event topology can basically be the same as the EWVB production from the leading orders. In result, tagging the Higgs boson from gluon fusion has been suffering from the irreducible backgrounds much more than the other subdominant productions including vector boson fusion (VBF), Higgsstrahlung (VH), and $t \bar{t} H$, as it does not have associated objects with fixed particle identity good for tagging the whole process.

For this reason, the most stringent constraints for probing the Yukawa couplings of Higgs bosons have usually been obtained via the non-ggH processes, e.g., in $H \rightarrow b \bar{b}$ [6], $c \bar{c}$ [7], $\tau^{+} \tau^{-}$[8], $\mu^{+} \mu^{-}$[9], and $e^{+} e^{-}$[10]. The same argument also applies to the searches of Higgs boson pair production via gluon fusion against the EWVB backgrounds, but in this case things can get worse, as the dominant ggH contribution increases (93\%).

In this paper, we revisit and generalize an overlooked property and investigate a new possibility for boosting Higgs boson searches via $\mathrm{ggH}$. We focus on the sizable differences in quark-gluon composition of the central initial state radiation (ISR) jets between the general ggH productions and their irreducible EWVB backgrounds. Based on such differences, we then show that tagging the central gluon jets from ISR can provide useful discrimination power to overall Higgs boson searches. The difference was stated earlier in Ref. [11] without attention, and the new possibility on the difference was claimed in Ref. [12] for 
$H \rightarrow \mu \mu$ and studied [13] for a monojet analysis. As the new method can have big impacts, here we generalize the property for (multiple) Higgs boson and EWVB production, emphasizing that, in the central region of detector, the leading ISR jet from ggH is mostly a gluon jet. To prove its experimental feasibility, then we apply the new method in search for invisible Higgs boson decays and show that the limit on the Higgs boson invisible decay branching ratio can be improved significantly $(60 \% \rightarrow 5 \%)$ for the most dominant gluon fusion, to be the most constraining channel, which has been not so useful compared to the other channels at the LHC.

This paper is composed as the following. In Sec. II, we discuss the dynamics of the ISR from two main processes, gluon fusion Higgs boson production and massive vector boson production, quantitatively at the leading order, and investigate the existence of a gluon-enriched kinematic region of leading ISR jet from the ggH process. The discussion is generalized by including the case of general Higgs boson signal production with $n$ Higgs boson $\left(\mathrm{ggH}^{n}+\right.$ jets $)$ and EWVB production $\left(V^{n}+\right.$ jets $)$. In Sec. III, we demonstrate the performance of the event classifier based on the ISR jet properties at detector level, in search for invisible Higgs boson decay. The performances of various discrimination models are compared in terms of the upper limit of confidence level on the invisible Higgs boson branching ratio in Sec. IV, and Sec. V is devoted to the conclusion. Miscellaneous details about multivariate models using deep neural networks and the others are added in the Appendixes.

\section{DYNAMICS OF LEADING ISR JET}

In this section, we discuss the dynamics of ISR at leading order associated with the ggH and EWVB production at the LHC. Figure 1 shows the leading diagrams (in bold) of general Higgs boson signal production from gluon fusion $\left(\mathrm{ggH}^{n}+\right.$ jets $)$ and EWVB production $\left(V^{n}+\right.$ jets $)$ as irreducible backgrounds with an emission of ISR(s), for three different initial parton configurations $(g q, g g$, and $q \bar{q})$.

\begin{tabular}{lccc}
\hline Proc. & $g q$ & $g g$ & $q \bar{q}$ \\
\hline $\mathrm{V}^{n}+\mathrm{j}$ & & & \\
& & & \\
& & &
\end{tabular}

FIG. 1. Leading diagrams (bold) of the (multi-)Higgs boson production from gluon fusion $\left(\mathrm{ggH}^{n}+\right.$ jets), against the corresponding irreducible (multi-)EWVB backgrounds $\left(V^{n}+\right.$ jets) with additional ISR(s) for three parton initial states $(g q, g g$, and $q \bar{q})$.
Here, the $n$ can be larger than one for multi-Higgs boson or multi-EWVB production, and additional Higgs boson or EWVB production with $n \geq 2$ is also represented. The gluon lines in gray indicate extra gluon emissions, and the $V^{n}+$ jets diagram (bottom center) from $g g$ initial states is drawn also in gray, as it is subleading to the other five diagrams in $\alpha_{s}$. By the irreducibility, we can assume that the particle IDs from the decays of the Higgs boson and EWVB are the same or very similar.

From Fig. 1, it should be first noted that the flavor of ISR(s) emitted in the leading diagrams (in bold) is uniquely fixed, since the other final state $\left(H^{n} / V^{n}\right)$ accompanied with is a colorless non-QCD particle in the 2 to $(n+1)$ processes, for the given initial parton configurations. It is also noticeable that the parton luminosity functions, $\mathcal{L}_{g q, g g, q \bar{q}}$ referring to Fig. 2(a) for the three initial partonic states, are hierarchical- $\mathcal{L}_{g q}>\mathcal{L}_{g g} \gg \mathcal{L}_{q \bar{q}}$, e.g., $\mathcal{L}_{g q}: \mathcal{L}_{g g}: \mathcal{L}_{q \bar{q}} \sim 2: 1: 0.07$ at $\sqrt{\hat{s}} \sim 100 \mathrm{GeV}$-and such a hierarchy persists to higher energy scale as shown in the same plot. Based on these two observations, the dominant flavor of the leading ISR jet from the whole $\mathrm{ggH}^{n}+$ jets and $V^{n}+$ jets processes can be predicted and their quarkgluon compositions can be compared.

Let us consider the background process, the production of EWVB, first. In the $n=1$ case, the differential partonic cross sections in the center-of-mass frame with respect to Mandelstam variable $\hat{t}$ are given as the following for the respective processes, $q \bar{q} \rightarrow V g$ and $g q \rightarrow V q$ :

$$
\begin{aligned}
& \frac{d \hat{\sigma}_{V g}}{d \hat{t}}=\frac{\alpha_{3}}{16 \hat{s}^{2}} \frac{C_{F}}{N_{c}} \sum_{q}\left(g_{V}^{q 2}+g_{A}^{q}\right) \frac{\hat{t}^{2}+\hat{u}^{2}+2 \hat{s} m_{V}^{2}}{\hat{t} \hat{u}}, \\
& \frac{d \hat{\sigma}_{V q}}{d \hat{t}}=\frac{\alpha_{3}}{16 \hat{s}^{2}} \frac{T_{F}}{N_{c}} \sum_{q}\left(g_{V}^{q 2}+g_{V}^{q}{ }^{2}\right)\left(-\frac{\hat{s}^{2}+\hat{u}^{2}+2 \hat{t} m_{V}^{2}}{\hat{s} \hat{u}}\right),
\end{aligned}
$$

with the number of colors $N_{c}$, the mass of gauge boson $m_{V}$, strong coupling $\alpha_{3}$, vectorial coupling $g_{V}^{q}$, and axial coupling $g_{A}^{q}$ between $V$ and massless quarks $q$, so, e.g., for the Z-boson case, $g_{V}^{q}=\frac{g_{2}}{\cos \theta_{W}}\left(\frac{1}{2} T_{3}^{q}-Q^{q} \sin ^{2} \theta_{W}\right)$ and $g_{A}^{q}=\frac{g_{2}}{2 \cos \theta_{W}} T_{3}^{q}$. The $T_{F}(=1 / 2)$ and $C_{F}(=4 / 3)$ are the Dynkin index and quadratic Casimir of fundamental representation of QCD, respectively. Since the matrix elements corresponding to the cross sections have crossing symmetry with each other, their angular profiles are not so distinctive in the LAB frame even after the integration with parton distribution functions as can be seen in Fig. 2(b). However, their total cross sections become very different considered with the two main scaling factors: (i) different interaction strengths from different initial states and (ii) hierarchical initial parton luminosity function values. As the $q \bar{q} \rightarrow V g$ process is averaged over two quarks, so the amplitude has a $\operatorname{tr}\left(t_{a} t^{a}\right) / N_{c}^{2}=C_{F} / N_{c}$ factor with $t_{a}$ as the $S U(3)_{c}$ generators at fundamental representation, while the $g q \rightarrow V q$ process has $\operatorname{tr}\left(t_{a} t^{a}\right) /\left(N_{c}\left(N_{c}^{2}-1\right)\right)=T_{F} / N_{c}$. 

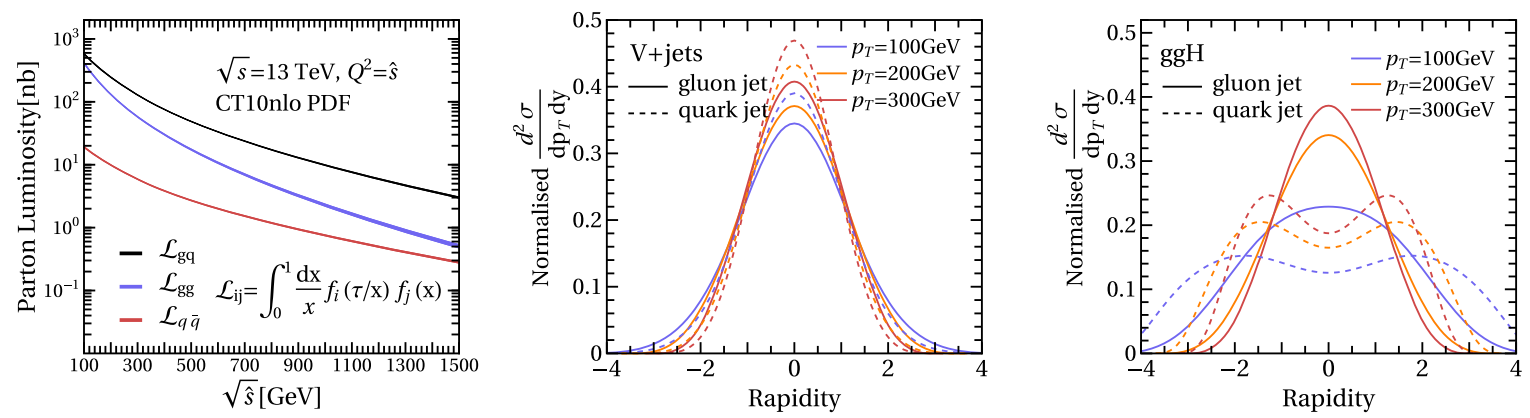

FIG. 2. (a) The parton luminosity $\mathcal{L}_{i j}$ with parton density function (PDF) $f_{i}\left(x, Q^{2}=\hat{s}\right)$ at the LHC with CT10nlo PDF as indicated in the figure. (b) Rapidity distribution of leading gluon and quark ISR jets from $Z$ boson production and (c) from ggH processes.

Although $T_{F}<C_{F}$, the hierarchically larger parton luminosity function of $g q, \mathcal{L}(g q)$ compared to $\mathcal{L}(q \bar{q})$, makes the hadronic total cross section $\sigma(p p \rightarrow V q)$ much bigger than the $\sigma(p p \rightarrow V g)$ by $\mathcal{O}(10)$ at the LHC.

This property can be generalized also for $V^{n}(n \geq 2)+$ jets processes, and in result the leading ISR jet is expected most likely to be a quark jet for general EWVB processes. As a demonstration of the quark jet portion dominance, we checked gluon jet portions $R^{g}$ of the leading ISR $\left(\left|\eta^{j_{1}}\right| \leq 1\right.$ and $p_{T}^{j_{1}}>100 \mathrm{GeV}$ ) in various EWVB processes, and they are found to be (i) $R_{(W W, W Z, Z Z)}^{g} \approx(0.20,0.16,0.30)$ for $V^{n=2}+$ jets processes, (ii) $R_{(W, Z)}^{g} \approx(0.13,0.19)$ for $V^{n=1}+$ jets, and (iii) $R_{\gamma \gamma}^{g} \approx 0.15$ for prompt diphoton + jet processes, as in Fig. 3 .

For the ggH signal, since $\mathcal{L}_{q \bar{q}}$ is much smaller than the others, the $q \bar{q} \rightarrow H g$ process can be ignored for the estimation. Relevant partonic differential cross sections, $d \hat{\sigma}_{H q} / d \hat{t}$ from $g q \rightarrow H q$ and $\hat{\sigma}_{H g} / d \hat{t}$ from the $g g \rightarrow H g$ process, computed at next-to-leading order (NLO) with massless five quarks and a finite top-mass effect, are given [5] as the following:

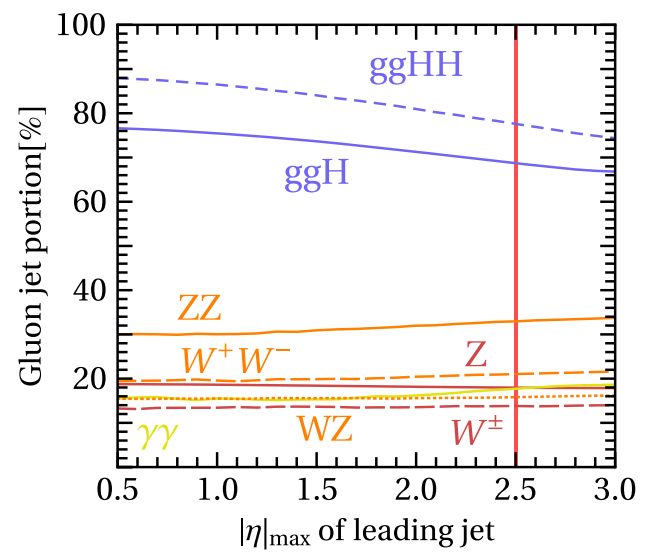

FIG. 3. Gluon portion over $\left|\eta^{j_{1}}\right|_{\max }\left(p_{T}^{j_{1}}>100 \mathrm{GeV}\right)$ of the leading ISR jet associated with $\mathrm{ggH}^{n}$ and $V^{n}$ production.

$$
\begin{aligned}
\frac{d \hat{\sigma}_{H g}}{d \hat{t}}= & \frac{\alpha_{3}^{3}}{16 \pi^{2} \hat{s}^{2} v^{2}} \frac{C_{A}}{N_{c}^{2}-1} \frac{m_{H}^{8}}{\hat{s} \hat{t} \hat{u}}\left(\left|A_{2}(\hat{s}, \hat{t}, \hat{u})\right|^{2}\right. \\
& \left.+\left|A_{2}(\hat{t}, \hat{u}, \hat{s})\right|^{2}+\left|A_{2}(\hat{u}, \hat{s}, \hat{t})\right|^{2}+\left|A_{4}(\hat{s}, \hat{t}, \hat{u})\right|^{2}\right), \\
\frac{d \hat{\sigma}_{H q}}{d \hat{t}} & =\frac{\alpha_{3}^{3}}{64 \pi^{2} \hat{s}^{2} v^{2}} \frac{C_{F}}{N_{c}^{2}-1}\left(-\frac{\hat{s}^{2}+\hat{u}^{2}}{\hat{t}}\right) \frac{m_{H}^{4}\left|A_{5}(\hat{t}, \hat{s}, \hat{u})\right|^{2}}{(\hat{s}+\hat{u})^{2}} .
\end{aligned}
$$

Here $m_{H}$ is the mass of the Higgs boson, and $C_{A}$ is the quadratic Casimir of adjoint representation of $S U(3)_{c}$ so that $C_{A}=N_{c}^{2}-1$ is $8 . A_{2}, A_{4}$, and $A_{5}$ are loop functions of which definitions are in Appendix A. From $\mathcal{L}_{g g}<\mathcal{L}_{g q}$, one may think that the hadronic cross section $\sigma(p p \rightarrow H q)$ is larger than the other one, $\sigma(p p \rightarrow H g)$, but there is a difference in associated color factors. It makes $C_{A} / C_{F}=$ $9 / 4$ enhancement on $\sigma(p p \rightarrow H g)$, and two cross sections are compatible with each other. Therefore, the quark-gluon portion of the leading ISR jet can highly depend on the dynamics of the leading ISR from the two dominant signal processes, especially on their transverse momentum and angular distributions.

To understand the differences on the $p_{T}$ profiles of the two processes, let us consider the infinite-topmass $\left(m_{t} \rightarrow \infty\right)$ limit. This limit is safe as long as $\left|\hat{s}-m_{H}^{2}\right| /\left(4 m_{t}^{2}\right) \lesssim 1$, with the mass of the top quark, $m_{t}$. In this limit, loop functions are approximated as

$$
A_{2}(\hat{s}, \hat{t}, \hat{u}) \rightarrow-\frac{\hat{s}^{2}}{3 m_{H}^{4}}, \quad A_{4} \rightarrow-\frac{1}{3},
$$

and

$$
A_{5}(\hat{s}, \hat{t}, \hat{u}) \rightarrow \frac{2}{3} \frac{\hat{t}+\hat{u}}{m_{H}^{2}},
$$

also for all the other permuted arguments. The effective differential cross sections $d \hat{\sigma}_{H g}^{\mathrm{HEFT}} / d \hat{t}$ and $d \hat{\sigma}_{H q}^{\mathrm{HEFT}} / d \hat{t}$ are, respectively, 


$$
\begin{aligned}
& \frac{d \hat{\sigma}_{H g}^{\mathrm{HEFT}}}{d \hat{t}}=\frac{\alpha_{3}^{3}}{144 \pi^{2} \hat{s}^{2} v^{2}} \frac{C_{A}}{N_{c}^{2}-1} \frac{\hat{s}^{4}+\hat{t}^{4}+\hat{u}^{4}+m_{H}^{8}}{\hat{s} \hat{t} \hat{u}} \\
& \frac{d \hat{\sigma}_{H q}^{\mathrm{HEFT}}}{d \hat{t}}=\frac{\alpha_{3}^{3}}{144 \pi^{2} \hat{s}^{2} v^{2}} \frac{C_{F}}{N_{c}^{2}-1}\left(-\frac{\hat{s}^{2}+\hat{u}^{2}}{\hat{t}}\right) .
\end{aligned}
$$

Note that each Mandelstam variable at the lowest order of transverse momentum $p_{T}$ is given by $\left(\hat{s} \simeq m_{H}^{2}\right)$

$$
\hat{t} \simeq m_{H} p_{T} e^{\Delta y} \quad \text { and } \quad \hat{u} \simeq m_{H} p_{T} e^{-\Delta y},
$$

where $\Delta y \equiv y_{b}-y_{j}$ is the rapidity difference of the two initial parton system $\left(y_{b}\right)$ and the jet $\left(y_{j}\right)$ in the LAB frame. Therefore,

$$
\frac{d \hat{\sigma}_{H g}^{\mathrm{HEFT}}}{d \hat{t}} \propto \frac{1}{p_{T}^{2}} \quad \text { and } \quad \frac{d \hat{\sigma}_{H q}^{\mathrm{HEFT}}}{d \hat{t}} \propto \frac{1}{p_{T}},
$$

which implies that a gluonic leading ISR jet is likely to have softer $p_{T}$ than a quark ISR jet.

The angular dependence can also be figured out by considering $s$-wave scattering of two processes. If the orbital angular momentum is zero, the spin of the outgoing gluon should be aligned along the direction perpendicular to the beam; otherwise, the total angular momentum is not conserved. However, the outgoing quark is likely to be backward to the incoming quark for angular momentum conservation in $g q \rightarrow H q$. Then, combined with the balanced momentum profile of the initial $g g$ state at the LHC, the gluon jets are likely to be emitted more in a central rapidity region with soft $p_{T}$, in comparison to the quark jet as can be seen in Fig. 2(c).

This property can also be applied to $\operatorname{ggH}^{n}(n \geq 2)+$ jets processes, and in result the leading ISR jet is expected likely to be a gluon jet for general ggH processes. As a demonstration of the gluon jet portion dominance, we also showed the gluon jet portions $R^{g}$ of the leading ISR $\left(\left|\eta^{j_{1}}\right| \leq 1\right.$ and $\left.p_{T}^{j_{1}}>100 \mathrm{GeV}\right)$, associated with a single $(\mathrm{ggH})$ and a pair of Higgs boson ( $\mathrm{ggHH})$ production from gluon fusion. They are found to be (i) $R_{\mathrm{ggH}}^{g} \approx 0.75$ for $\mathrm{ggH}^{n=1}+$ jets and (ii) $R_{\mathrm{ggHH}}^{g} \approx 0.87$ for a pair of Higgs boson production, as in Fig. 3. If $P_{T}$ cut is lowered to $P_{T}^{j_{1}}>50 \mathrm{GeV}$, the $R^{g}$ for the $\mathrm{ggH}^{n}\left(V^{n}\right)$ process increases (decreases), respectively, by $\sim 2 \%-5 \%$ in the $\left|\eta^{j_{1}}\right|_{\max }$ range of Fig. 3, which is also consistent with our expectation of the soft $p_{T}$ dominance of gluon ISR from the $\mathrm{ggH}$ processes, as in Eq. (4).

Like the two quark jets in the forward region from the $\mathrm{VBF}$, and the extra $Z$ or $W$ from the VH, now the $\mathrm{ggH}^{n}+$ jets also has such a unique property-the gluonlike ISR jets in the central region. In this regard, if some relevant techniques using quark-gluon tagging of ISR jets are employed, one can improve the constraints from the most dominant gluon fusion channel for a broad range of Higgs boson signatures which are buried in the irreducible EWVB backgrounds matched with.

\section{INVISIBLE DECAY OF HIGGS BOSON}

In order to demonstrate experimental feasibility, here we utilize the gluonic ISR jet from the $\mathrm{ggH}$ for constraining invisible decays of the Higgs boson where the decayed particles from the Higgs boson and irreducible EWVB are electrically neutral and invisible. Historically, there had been lots of studies on the possibility of invisible Higgs boson decays along the developments of the Standard Model and beyond. The early proposals include the models in diversity, e.g., with Majorons [14,15], supersymmetries [16], heavy neutrinos with radiatively generated masses [17], large extra dimensions $[18,19]$, the fourth generation [20-22], and so on, while recent interpretations are mainly based on the effective singlet extensions of the SM, in the context of socalled Higgs-portal models with dark matter [23-26]. For these proposals, there exist numerous phenomenological and experimental researches in the search for the invisible Higgs boson decays, via the production channels, including

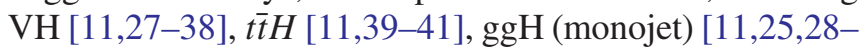
$30,34,35,42-44]$, and VBF [28,30,31,34,36-38,45-48], among which the VBF has been presented the most sensitive limits on the invisible Higgs boson decay BR at the LHC. There also have been interesting surveys via diffractive Higgs boson production [21], total decay width [49], Higgs boson rare B decays [50], di-Higgs boson [51], Higgs boson off-shell decays [52], and lepton colliders [53], including global analysis [54]. Throughout the searches, the dominant $\mathrm{ggH}$ channel has never been competitive to the other subdominant channels. However, employing the new method, we show that the most stringent constraint can be obtained from the ggH channel for the invisible Higgs boson decays, as is demonstrated in the next paragraphs.

Assuming the Higgs boson production cross section of the SM, we perform the analysis in search for the invisible Higgs boson decays in $E_{T}^{\text {miss }}+$ jets signature via the $\mathrm{ggH}+$ jets channel. Samples are generated by Monte Carlo simulated pp collisions at a center-of-mass energy of $\sqrt{s}=13 \mathrm{TeV}$ at the LHC, for $36 \mathrm{fb}^{-1}$ using MadGraph5 aMC@NLO v2.6.2 [55] interfaced with PYTHIA v8.235 [56] for hadronization and fragmentation. Delphes v.3.4.1 is used for detector simulation [57]. The signal process $(\mathrm{ggH}+$ jets $)$ is generated with up to extra one jet at LO, taking into account finite top-mass effects [58] with $M_{H}=125 \mathrm{GeV}$, and backgrounds are generated at NLO in QCD. We use the FxFx scheme with a $k_{T}$ algorithm and $\Delta R=1$ for jet merging [59]. For jet clustering, FastJet v3.2.1 [60] is used with an anti- $k_{T}$ algorithm with $\Delta R=0.4$, and CT10NLO [61] is used for the parton distribution function.

Among the relevant background processes- $V(Z(\nu \nu)$, $W(\ell \bar{\nu}))+$ jets, diboson, top quarks, $Z / \gamma \rightarrow \ell \bar{\ell}$, and QCD multijets, where the leptons $(\ell)$ in $W / Z / \gamma$ decays are misidentified-we included only the most dominant 
irreducible EWVB backgrounds, $V+$ jets, while the others take just $O(1) \%$ level for the event selection criteria as follows [34]:

(i) $p_{T}^{j_{1}}>100 \mathrm{GeV}, \quad\left|\eta^{j_{1}}\right|<2.5, \quad E_{T}^{\text {miss }}>200 \mathrm{GeV}$, $\min _{j \in\{\text { jets }\}} \Delta \phi\left(\vec{p}_{T}^{\text {miss }}, \vec{p}_{T}^{j}\right) \geq 0.5$, and $N_{\text {jet }} \geq 1$.

The first (second) cut on the transverse momentum (pseudorapidity) of the leading jet is imposed to suppress all of the backgrounds, the third cut on the missing transverse energy is mainly to reduce the QCD and top quarks, and the fourth cut with the missing transverse momentum $\vec{p}_{T}^{\text {miss }}$ suppresses the QCD multijets very efficiently [35].

There also exist contributions from other Higgs boson productions, $\mathrm{VBF}$ and $\mathrm{VH}$ with yield rates (ggH:VBF: VH 70:20:10\%). However as the leading jets from VBF are most likely quark jets opposed to the gluonic leading jets in the $\mathrm{ggH}+$ jets, we checked that the VBF can be easily separated (see Appendix C) from the $\mathrm{ggH}+$ jets by tagging the gluonic central leading jet in addition to the forward jet tagging for VBF. As for the VH which also has quark-jet-like leading ISRs according to the same argument as the $V+$ jets, it can have additional selection criteria $[62,63]$ for identifying jets from hadronically decaying vector bosons. In this regard, to demonstrate the main idea without making the event selection scheme too complicated, we simply consider the $\mathrm{ggH}+$ jets as the only signal versus the $V+$ jets as the main background in this analysis, without loss of consistency in applying the flavor information for discrimination of a gluon-jet-rich $\mathrm{ggH}$ signal from general quark-jet-rich backgrounds.

\section{MULTIVARIATE ANALYSIS AND RESULT}

We use a set of jet substructure variables [64], say, $S^{\text {jet }}$, in our analysis as the following:

(i) $S^{\mathrm{jet}} \equiv\left\{n_{\mathrm{tk}}\right.$ (track multiplicity) [65], girth $[65,66]$, broadening [67], energy-energy correlation (EEC) [68] with $\beta=0.2$ [69], and rms- $\left.p_{T}[65]\right\}$, which contain the information on jet flavors. It can also be extended to include more raw data, e.g., jet images [70,71] for deep learning. Among the five jet substructure variables used, the girth as the linear radial moment of a jet reflects a fatness or radius of a jet. As gluon jets tend to have more showers and be fatter by the color factor enhancement,
$C_{A}(g \rightarrow g g) / C_{F}(q \rightarrow g q)$, such a property can be checked in the girth distribution of the leading jet from $\mathrm{ggH}$ and $V+$ jet processes in Fig. 4(b).

Jet substructure observables have been used to build a jet tagger, $\mathrm{P}_{q / g}\left(S^{\mathrm{jet}}\right)$, while the kinematic observables, such as reconstructed four-momenta of jets, have been used to build an event classifier, $\mathrm{P}_{\mathrm{S} / \mathrm{B}}\left(\left\{p^{\text {jet }}, \ldots\right\}\right)$. However, as can be seen from $d^{2} \sigma / d p_{T}^{\text {jet }} d y^{\text {jet }}$ in Fig. 2(c), the flavor of a jet can have a correlation with kinematic information depending on the scattering process. This observation motivates us to build $\mathrm{P}_{\mathrm{S} / \mathrm{B}}\left(\left\{p^{\mathrm{jet}}, \ldots\right\} \cup S^{\mathrm{jet}}\right)$ rather than a factorized classifier, $\mathrm{P}_{\mathrm{S} / \mathrm{B}}\left(\left\{p^{\text {jet }}, \ldots\right\}\right) \otimes \mathrm{P}_{q / g}\left(S^{\mathrm{jet}}\right)$.

For the $\mathrm{S}(\mathrm{B})=\operatorname{ggH}(V+$ jets $)$ process, Fig. 4 shows the normalized distribution of (a) $E_{T}^{\text {miss }}$, (b) girth, (c) $\mathrm{P}_{\mathrm{S} / \mathrm{B}}\left(S^{\text {jet }}\right)$, and (d) $\mathrm{P}_{\mathrm{S} / \mathrm{B}}\left(S^{\text {jet }} \cup\left\{E_{T}^{\text {miss }}, p_{T}^{\text {jet }}, \eta^{\text {jet }}\right\}\right)$. The two event classifiers $\mathrm{P}_{\mathrm{S} / \mathrm{B}}$ in Figs. 4(c) and 4(d) are obtained by training neural networks with 2-4 layers, each with 200300 nodes with the specified input features. We used one million event samples with Keras [72] for building and training the neural network models. More detailed information about using the neural network can be found in Appendix. B. It is noticeable that the event classifier using the set of jet substructures alone can provide much better separation of signal and background compared to the one $E_{T}^{\text {miss }}$ as in Fig. 4(c). Combining them all, we get the best separation, as is clearly seen in Fig. 4(d).

The result obtained up to now can be used to discover invisible Higgs boson decay or put constraints on the invisible branching ratio of the Higgs boson. After selecting the events with the criteria, we performed the profile likelihood ratio test following the procedure in Ref. [73] with the four template distributions in Fig. 4. The likelihood function is given as

$$
\mathcal{L}=\prod_{i=1}^{N_{\text {bin }}} \frac{\hat{n}_{i}^{n_{i}}}{n_{i} !} e^{-\hat{n}_{i}} \times \frac{1}{2 \pi} e^{-(1 / 2)\left(\theta_{s}^{2}+\theta_{b}^{2}\right)},
$$

where $n_{i}$ is the number of events (or pseudoevents) in the $i$ th bin and $\hat{n}_{i}$ is the number of expected events with branching ratio parameter $\mu=\frac{\sigma}{\sigma_{\mathrm{SM}}} \times \mathrm{BR}(h \rightarrow$ inv) (production cross section of invisibly decaying Higgs boson
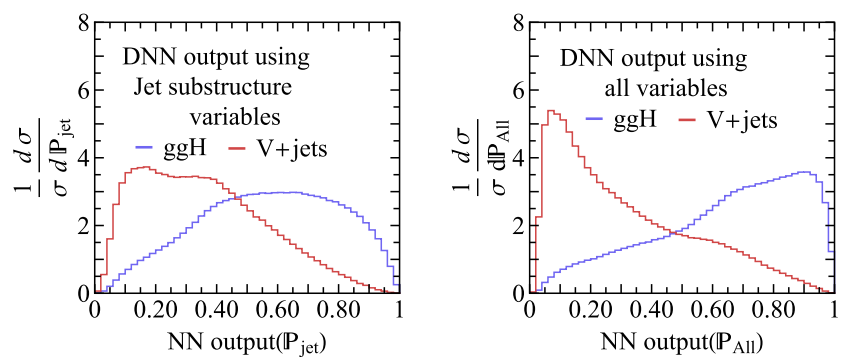

FIG. 4. Signal and background profiles in various templates. (a) $E_{T}^{\text {miss }}$, (b) girth of the leading jet, (c) event classifier $\mathrm{P}_{\mathrm{S} / \mathrm{B}}\left(S^{\mathrm{jet}}\right)$ (1, ggH-like; $0, V+$ jet) trained using the jet substructure observables $S^{\text {jet }}$, and $(d) \mathrm{P}_{\mathrm{S} / \mathrm{B}}\left(S^{\text {jet }} \cup\{\operatorname{kin}\}\right)$ using all features. 
over the total cross section of the SM Higgs boson, $\sigma_{\mathrm{SM}}$ ), i.e.,

$$
\hat{n}_{i}=\mu N_{s} P_{s}(i)\left(1+f_{s}\right)^{\theta_{s}}+N_{b} P_{b}(i)\left(1+f_{b}\right)^{\theta_{b}} .
$$

Here the $P_{s(b)}(i)$ is the expected event rate in the $i$ th bin, given the total number of events $N_{s(b)}$ survived the cut, and the $\theta_{s(b)}$ in the Poisson and prior probabilities denotes a nuisance parameter associated to the systematic uncertainty $f_{s(b)}$ of signal (background). As a global variation of event rates in signal and background distributions, we tested the $f_{s(b)}$ in 5\%-20\% [34], which changes the median of expected upper limit $3 \%$ at most and set $f_{s(b)}$ to $10 \%$. The signal cross section $(\mathrm{ggH}+\mathrm{X})$ is taken from Ref. [74] computed at NNLO + NNLL QCD and NLO EW, and we applied the efficiency on the selection criteria evaluated using simulated event samples, for the fiducial signal yield. For the background process $(V+$ jets), we take both the cross section and efficiency from our MC simulation of the $Z+$ jets, and the fiducial background yield was obtained by a $\mathrm{K}$ factor (1.53) with respect to the $Z+$ jet, to take the $W+$ jets into account simply, reproducing the expected limit using missing transverse energy [35].

For the four profile likelihood ratio tests with and without the new features of jet flavors ("missing $E_{T}$," "girth," "jet substructure variables," and "all variables"), we obtained the upper limits on the branching ratio of invisible Higgs boson decays in $95 \%$ of confidence level, for the integrated luminosity $36 \mathrm{fb}^{-1}$ at the LHC, as in Fig. 5 (right panel), and show them with the existing experimental results (left panel) [48]—ggH-tag, VBF-tag, and combined, which did not use jet flavor information. As the results in the two red-boxed columns can directly be compared under the same features and selection criteria, we summarize our results in Table I with the errors rescaled by the correction

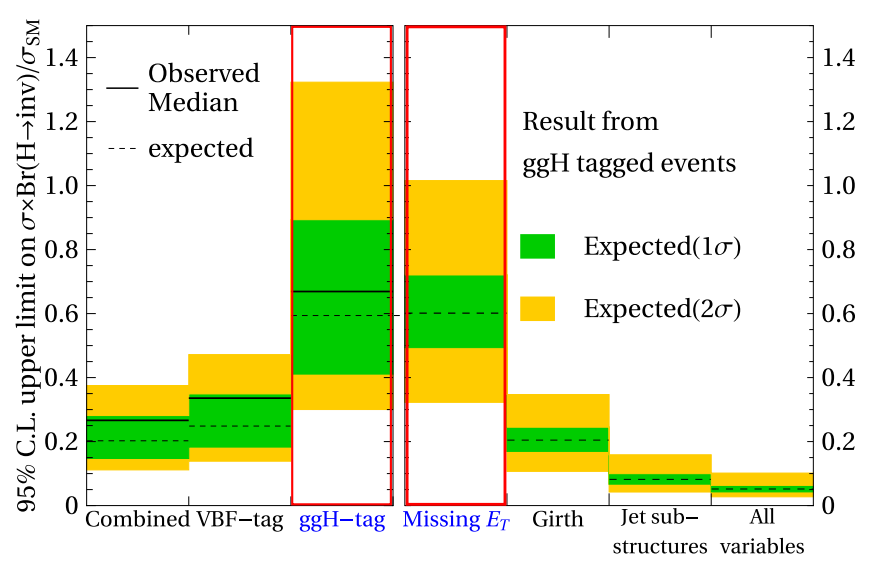

FIG. 5. Upper limit in $95 \%$ of confidence level (C.L.) on $\frac{\sigma \times \operatorname{Br}(H \rightarrow \text { inv })}{\sigma_{\mathrm{SM}}}$ with the integrated luminosity $36 \mathrm{fb}^{-1}$. Here we used 5000 ensembles of a pseudo dataset which consists of background events only. The left panel shows the results from the experiment [48].
TABLE I. Summary on the upper limits in $95 \%$ of confidence level on $\frac{\sigma \times \mathrm{BR}(\mathrm{H} \rightarrow \mathrm{inv})}{\sigma_{\mathrm{SM}}}$ for $36 \mathrm{fb}^{-1}$ at the LHC, for the four template distributions in Fig. 4. The errors were rescaled by the factors projecting the "missing ET" band to the reference "ggH-tag" band in Fig. 5.

\begin{tabular}{lcccc}
\hline \hline $\mathcal{L}$ & $E_{T}^{\text {miss }}$ & Girth & DNN (jet sub) & DNN (all) \\
\hline $36 \mathrm{fb}^{-1}$ & $60.2_{-18.3}^{+30.0} \%$ & $20.4_{-5.99}^{+10.1} \%$ & $8.3_{-2.55}^{+4.46} \%$ & $5.2_{-1.54}^{+2.83} \%$ \\
\hline \hline
\end{tabular}

factors projecting the obtained missing $E_{T}$ band to the reference ggH-tag band. The result shows that the limit on Higgs boson invisible decays from the $\mathrm{ggH}$ can significantly be improved from $60 \%$ down to $5 \%$ if subjet-level information of the leading ISR is employed. It is interesting that the jet substructures alone provide stronger constraints (8\%) than the missing transverse energy in the Higgs boson invisible search. Moreover, combining the features in two kinds, we end up with the best sensitive result (5\%) only from the $\mathrm{ggH}$, much lower than the one obtained from $\operatorname{VBF}(\sim 20 \%)$.

Though more sophisticated understanding and treatment of systematic errors are necessary to obtain a firm number for the expected limit on the Higgs boson invisible decays, the exercise we did in this paper strongly suggests that $1 \%$ (2\%) precision for the Higgs boson invisible branching ratio at the end of the LHC running with $3 \mathrm{ab}^{-1}\left(300 \mathrm{fb}^{-1}\right)$ is a plausible expectation from gluon fusion solely. It is also expected that the limit can significantly be improved again if it is combined with the results from VBF and other processes.

\section{CONCLUSION}

We revisited and generalized the property - the gluon-rich leading ISR jets in the central rapidity region from gluon fusion Higgs boson production versus the quark-rich EWVB backgrounds - and proposed the idea to improve general Higgs boson searches produced from ggH by tagging the central gluonic ISR jets. Applying the new method to the searches of invisible decays of Higgs bosons, we showed that the $\mathrm{ggH}$ can be the best channel with the improved limit on invisible Higgs boson decay branching ratio $(60 \% \rightarrow 5 \%)$, significantly exceeding the best limit given by the other channels-VBF ( 20\%) and VH ( 40\%). The physics and methods in this analysis can also be applied to a broad range of new resonance and Higgs boson production induced by gluon fusion, e.g., in the search for exotic and rare Higgs boson decays and di-Higgs boson production, concurrently with their irreducible EWVB backgrounds mostly containing quark-jet-dominant ISRs.

\section{ACKNOWLEDGMENTS}

This work was supported by the National Research Foundation of Korea (NRF), Grant No. 2017R1A2B2010749. W. S. C. and D. L. were also 
supported by the NRF funded by the Ministry of Science and ICT, Grant No. 2017R1C1B2011048.

\section{APPENDIX A: LOOP FUNCTIONS}

Here we summarized the loop functions relevant for the Higgs boson production via gluon fusion with a jet at the leading order with finite top-quark mass. The definition and integral forms are referred to Ref. [5]. The two loop functions relevant for $g g \rightarrow H g, A_{2}(s, t, u)$ and $A_{4}(s, t, u)$, are defined, respectively, by

$$
\begin{aligned}
& A_{2}(s, t, u)=b_{2}(s, t, u)+b_{2}(s, u, t), \\
& A_{4}(s, t, u)=b_{4}(s, t, u)+b_{4}(t, u, s)+b_{4}(u, s, t) .
\end{aligned}
$$

Here $b_{2}$ and $b_{4}$ are defined by

$$
\begin{aligned}
b_{2}(s, t, u)= & \frac{m_{t}^{2}}{m_{H}^{4}}\left[\frac{s(u-s)}{u+s}+\frac{2 u t(u+2 s)}{(u+s)^{2}}\left(W_{1}(t)-W_{1}\left(m_{H}^{2}\right)\right)\right. \\
& +\left(m_{t}^{2}-\frac{1}{4} s\right)\left(\frac{1}{2} W_{2}(s)+\frac{1}{2} W_{2}\left(m_{H}^{2}\right)-W_{2}(t)+W_{3}\left(s, t, u, m_{H}^{2}\right)\right) \\
& +s^{2}\left(\frac{2 m_{t}^{2}}{(s+u)^{2}}-\frac{1}{2(s+u)}\right)\left(W_{2}(t)-W_{2}\left(m_{H}^{2}\right)\right) \\
& \left.+\frac{u t}{2 s}\left(W_{2}\left(m_{H}^{2}\right)-2 W_{2}(t)\right)+\frac{1}{8}\left(s-12 m_{t}^{2}-\frac{4 u t}{s}\right) W_{3}\left(t, s, u, m_{H}^{2}\right)\right], \\
b_{4}(s, t, u)= & \frac{m_{t}^{2}}{m_{H}^{2}}\left[-\frac{2}{3}+\left(\frac{m_{t}^{2}}{m_{H}^{2}}-\frac{1}{4}\right)\left(W_{2}(t)-W_{2}\left(m_{H}^{2}\right)+W_{3}\left(s, t, u, m_{H}^{2}\right)\right)\right],
\end{aligned}
$$

respectively, with $m_{t}$ the mass of the top quark and $m_{H}$ the mass of the Higgs boson. The other five light quarks are considered to be massless. Again, $W_{1}, W_{2}$, and $W_{3}$ can be defined as integral forms:

$$
\begin{aligned}
W_{1}(s) & =2+\int_{0}^{1} d x \ln \left(1-x(1-x) \frac{s}{m_{t}^{2}}-i \varepsilon\right), \\
W_{2}(s) & =2 \int_{0}^{1} \frac{d x}{x} \ln \left(1-x(1-x) \frac{s}{m_{t}^{2}}-i \varepsilon\right), \\
W_{3}(s, t, u, v) & =I_{3}(s, t, u, v)-I_{3}(s, t, u, s)-I_{3}(s, t, u, u), \\
I_{3}(s, t, u, v) & =\int_{0}^{1} d x\left(\frac{m_{t}^{2} t}{u s}+x(1-x)\right)^{-1} \ln \left(1-x(1-x) \frac{v}{m_{t}^{2}}-i \varepsilon\right) .
\end{aligned}
$$

The other form factor $A_{5}$ is as follows:

$$
A_{5}(s, t, u)=\frac{m_{t}^{2}}{m_{H}^{2}}\left[4+\frac{4 s}{t+u}\left(W_{1}(s)-W_{1}\left(m_{H}^{2}\right)\right)+\left(1-\frac{4 m_{t}^{2}}{t+u}\right)\left(W_{2}(s)-W_{2}\left(m_{H}^{2}\right)\right)\right] .
$$

Note that, in the main text, $A_{5}$ has $(\hat{t}, \hat{s}, \hat{u})$ as its argument rather than $(\hat{s}, \hat{t}, \hat{u})$. This is because the loop function $A_{5}$ was evaluated for $q \bar{q} \rightarrow H g$ rather than $g q \rightarrow H q$. Under the crossing symmetry, the differential cross section $d \hat{\sigma}_{q \bar{q} \rightarrow H g} / d \hat{t}$ of $q \bar{q} \rightarrow H g$ satisfies

$$
\frac{d \hat{\sigma}_{H q}}{d \hat{t}}(\hat{s}, \hat{t}, \hat{u})=-\frac{N_{c}}{N_{c}^{2}-1} \frac{d \hat{\sigma}_{q \bar{q} \rightarrow H g}}{d \hat{t}}(\hat{t}, \hat{s}, \hat{u}),
$$

and we used the same loop function with a different argument for the $g q \rightarrow H q$ process.

\section{APPENDIX B: DETAILS OF DEEP NEURAL NETWORK STRUCTURE}

The event classifiers which distinguish the ggH signal from $V+$ jets background are denoted as $\mathbf{P}_{\mathrm{S} / \mathrm{B}}\left(\mathbf{S}^{\text {jet }}\right)$ and $\mathbf{P}_{\mathrm{S} / \mathrm{B}}\left(\mathbf{S}^{\text {jet }} \cup\{\operatorname{kin}\}\right) . \mathrm{P}_{\mathrm{S} / \mathrm{B}}\left(\mathrm{S}^{\text {jet }}\right)$ uses five jet substructure variables, track multiplicity $\left(n_{\mathrm{tk}}[65]\right)$, girth $(G[65,66])$, broadening ( $B$ [67]), EEC $\left(C_{1}^{\beta}[68]\right)$ with $\beta=0.2$ [69], and rms- $p_{T}[65]$ of the jet. $\mathrm{P}_{\mathrm{S} / \mathrm{B}}\left(\mathrm{S}^{\text {jet }} \cup\{\operatorname{kin}\}\right)$ uses $E_{T}^{\text {miss }}, p_{T}^{\text {jet }}$, and $\eta_{T}^{\text {jet }}$ in addition to jet substructure variables used in $\mathrm{P}_{\mathrm{S} / \mathrm{B}}\left(\mathrm{S}^{\text {jet }}\right)$, so a total of eight variables are used. 
TABLE II. DNN model specification and training prescriptions used for this study.

\begin{tabular}{|c|c|c|}
\hline & $\mathbf{P}_{\mathrm{S} / \mathrm{B}}\left(\mathbf{S}^{\mathrm{jet}}\right)$ & $\mathbf{P}_{\mathrm{S} / \mathrm{B}}\left(\mathbf{S}^{\mathbf{j e t}} \cup\{\operatorname{kin}\}\right)$ \\
\hline Training data & \multicolumn{2}{|c|}{$1 \mathrm{M}(0.5 \mathrm{M}$ each for sig/bg) } \\
\hline Validation data & \multicolumn{2}{|c|}{$1 \mathrm{M}(0.5 \mathrm{M}$ each for $\mathrm{sig} / \mathrm{bg})$} \\
\hline Preprocessing & \multicolumn{2}{|c|}{ Standard scaler } \\
\hline NN package & \multicolumn{2}{|c|}{ Keras [72] with TensorFlow back end } \\
\hline NN structure & \multicolumn{2}{|c|}{ Fully connected feed-forwarding (FF) layers } \\
\hline Normalization & \multicolumn{2}{|c|}{ Batch normalization [75] } \\
\hline Drop out & $30 \%$ & $10 \%$ \\
\hline NN structure & \multicolumn{2}{|c|}{ Two hidden FF layers with 300 nodes each Four hidden FF layers with 200 nodes each } \\
\hline Optimizer & \multicolumn{2}{|c|}{ Adam [76] } \\
\hline Loss function & \multicolumn{2}{|c|}{ Categorical cross-entropy } \\
\hline Learning rate & 0.001 & 0.001 \\
\hline Minibatch size & \multicolumn{2}{|c|}{50000} \\
\hline Activation function & \multicolumn{2}{|c|}{ ReLU for intermediate layers, soft-max for output layer } \\
\hline Initialization & \multicolumn{2}{|c|}{$\mathrm{He}[77]$} \\
\hline
\end{tabular}

The definition of jet substructure variables are as the following:

$$
\text { girth: } G=\frac{1}{p_{T}^{\text {jet }}} \sum_{i \in\{\text { const }\}} p_{T}^{i}\left|\Delta \vec{r}_{i}\right|,
$$

broadening: $B=\frac{1}{\sum_{i}\left|\vec{p}^{i}\right|} \sum_{i}\left|\vec{p}^{i} \times \hat{p}^{\text {jet }}\right|$

$$
=\frac{1}{\sum_{i}\left|\vec{p}^{i}\right|} \sum_{i}\left|\vec{k}_{T}^{i}\right|
$$

$$
\begin{aligned}
& \mathrm{EEC}: C_{1}^{\beta}=\frac{1}{\left(\sum_{i} p_{T}^{i}\right)^{2}} \sum_{i<j} p_{T}^{i} p_{T}^{j}\left(\Delta R_{i j}\right)^{\beta}, \\
& \operatorname{rms}-p_{T}: \sqrt{\left\langle p_{T}^{2}\right\rangle}=\frac{1}{p_{T}^{\mathrm{jet}}} \sqrt{\frac{1}{n_{\mathrm{tk}}} \sum_{i}\left(p_{T}^{i}\right)^{2}}
\end{aligned}
$$

where $\{$ const $\}$ means the set of constituents of a jet.

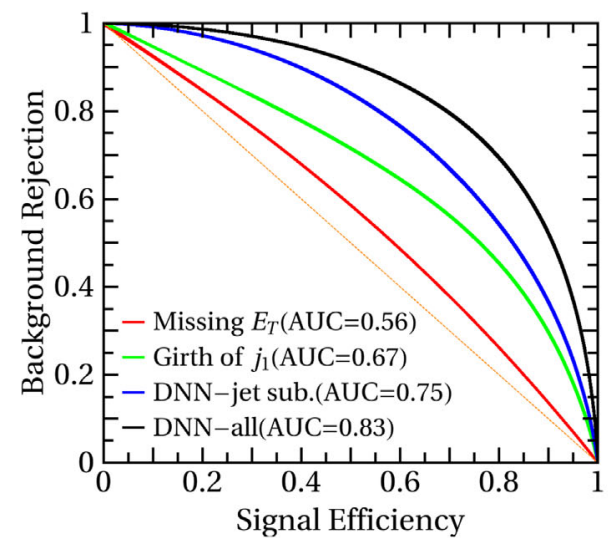

The specification of the neural network classifiers $\mathrm{P}_{\mathrm{S} / \mathrm{B}}\left(\mathrm{S}^{\mathrm{jet}}\right)$ and $\mathrm{P}_{\mathrm{S} / \mathrm{B}}\left(\mathrm{S}^{\mathrm{jet}} \cup\{\mathrm{kin}\}\right)$ is summarized in Table II especially on its structure, hyperparameters, and training prescriptions. In addition to the distribution of neural network outputs in the main text, here the receiver operating characteristic (ROC) curves and related $\epsilon_{\mathrm{sig}} / \sqrt{\epsilon_{\mathrm{bg}}}$ are shown in Fig. 6.

\section{APPENDIX C: SEPARATION OF VECTOR- BOSON-FUSION HIGGS BOSON PRODUCTION}

In this Appendix, we shortly discuss the discrimination of VBF Higgs boson production mechanism from another Higgs boson production mechanism, $\mathrm{ggH}$, and its background, Drell-Yan (DY) process. It is well known that the VBF process has characteristic two jets with large angular separation between them $\left(\left|\Delta \eta_{\mathrm{jj}}\right|\right)$ and a large invariant mass of them $\left(m_{\mathrm{jj}}\right)$. This handle is used to separate the VBF from the other processes, $\mathrm{ggH}$ and DY. On top of that, there is an additional handle, which is the parton contents of jets.

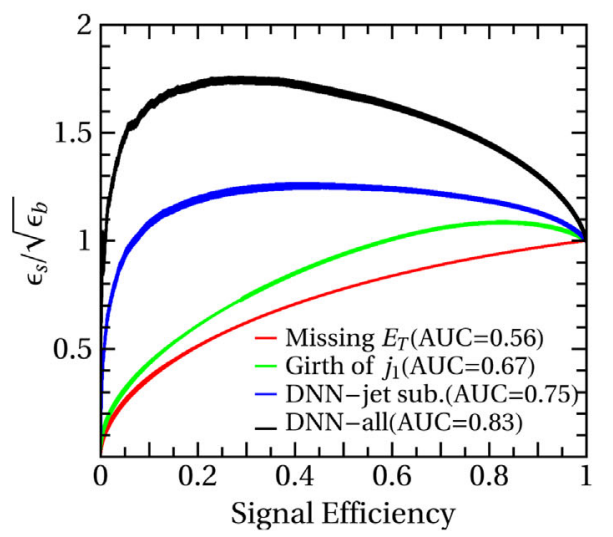

FIG. 6. ROC curves (left) and $\epsilon_{\mathrm{sig}} / \sqrt{\epsilon_{\mathrm{bg}}}$ curves (right) derived from respective ROC curves. In each panel, ROC derived from $E_{T}^{\mathrm{miss}}$ distribution is drawn with red, and the one from girth is green. $\mathrm{P}_{\mathrm{S} / \mathrm{B}}\left(\mathrm{S}^{\text {jet }}\right)$ and $\mathrm{P}_{\mathrm{S} / \mathrm{B}}\left(\mathrm{S}^{\text {jet }} \cup\{\right.$ kin $\left.\}\right)$ are drawn in blue and black, respectively. 

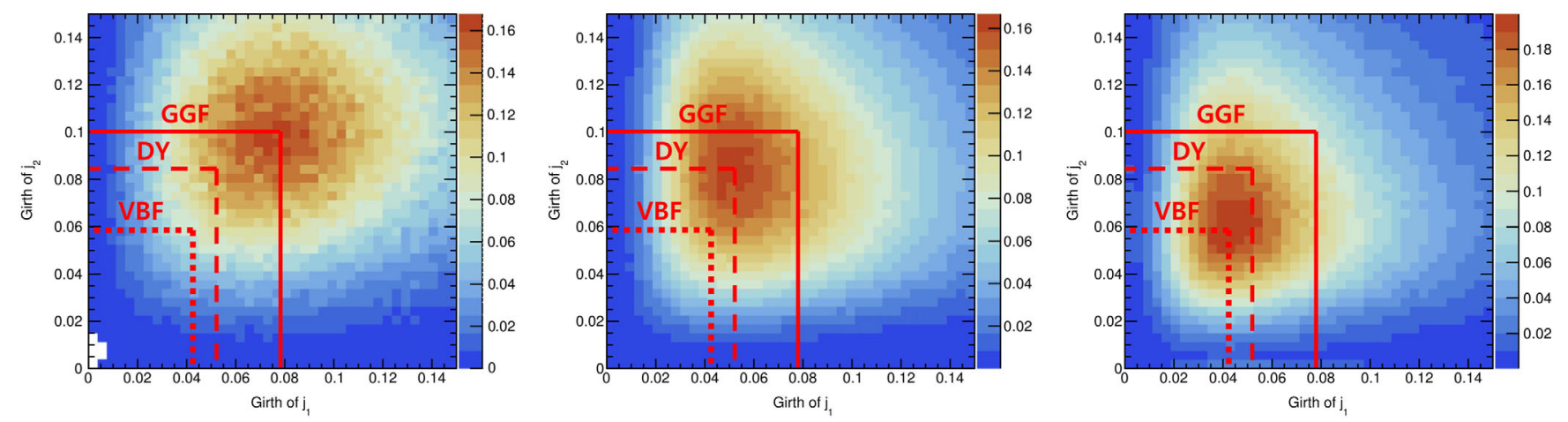

FIG. 7. The scatter plots of girth of leading jets ( $x$ axis) versus girth of subleading jets ( $y$ axis). The three plots came from different processes: ggH (left), DY (middle), and VBF (right). Red lines in plots indicate the peak position from three distributions. The solid red line points out the peak from the ggH process, and the dashed line for DY and dotted line for VBF. Details of event samples for this plot can be found in the text.

Note that the two jets of VBF processes are mostly quark jets, while the leading jets of $\mathrm{ggH}$ in the central region are mainly gluonic. Focusing on the leading jet flavor, therefore, does separate the VBF process from the ggH process. At the same time, the subleading jets from the DY process are more likely to be gluonic, due to parton luminosity. Hence, the VBF process can be also separated from the DY process by observing the parton contents of the subleading jets.

The difference in parton contents of associated jets can be seen in the two-dimensional distribution of leading jet girth and subleading jet girth as in Fig. 7. The event samples generated at $14 \mathrm{TeV}$ with aMC@NLO [55] at NLO in QCD with $m_{t} \rightarrow \infty$ limit are used for this plot. CT10NLO [61] PDF and Higgs characterization [78] model are used for the simulation. Events are showered using PYTHIA8 [56] and merged via the FxFx [59] scheme with $Q_{\text {cut }}=40 \mathrm{GeV}$. The jet clustering is done with an anti- $k_{T}$ algorithm with $\Delta R=0.4$ using FastJet [60], for $p_{T}^{\text {jet }} \geq 30 \mathrm{GeV}$. The fast detector simulation is done with Delphes [57].

In Fig. 7, the peaks of two-dimensional girth distributions from each process are placed away from the peaks of the others due to different quark or gluon composition of corresponding jets as we expected.
[1] F. Englert and R. Brout, Broken Symmetry and the Mass of Gauge Vector Mesons, Phys. Rev. Lett. 13, 321 (1964); P. W. Higgs, Broken symmetries, massless particles and gauge fields, Phys. Lett. 12, 132 (1964); Broken Symmetries and the Masses of Gauge Bosons, Phys. Rev. Lett. 13, 508 (1964).

[2] G. Aad et al. (ATLAS Collaboration), Observation of a new particle in the search for the Standard Model Higgs boson with the ATLAS detector at the LHC, Phys. Lett. B 716, 1 (2012); S. Chatrchyan et al. (CMS Collaboration), Observation of a new boson at a mass of $125 \mathrm{GeV}$ with the CMS experiment at the LHC, Phys. Lett. B 716, 30 (2012); Observation of a New Boson with Mass Near $125 \mathrm{GeV}$ in p p Collisions at $\sqrt{s}=7$ and $8 \mathrm{TeV}$, J. High Energy Phys. 06 (2013) 081.

[3] K. Cheung, J.S. Lee, and P.-Y. Tseng, Higgs precision (Higgcision) era begins, J. High Energy Phys. 05 (2013) 134; M. Cepeda et al., Report from Working Group 2: Higgs Physics at the HL-LHC and HE-LHC, CERN Yellow Reports: Monographs Vol. 7 (2019), pp. 221-584; J. de Blas et al., Higgs Boson studies at future particle colliders, J. High Energy Phys. 01 (2020) 139.
[4] H. Georgi, S. Glashow, M. Machacek, and D. V. Nanopoulos, Higgs Bosons from Two-Gluon Annihilation in ProtonProton Collisions, Phys. Rev. Lett. 40, 692 (1978).

[5] R. Ellis, I. Hinchliffe, M. Soldate, and J. van der Bij, Higgs decay to $\tau^{+} \tau^{-}$A possible signature of intermediate mass Higgs bosons at high energy hadron colliders, Nucl. Phys. B297, 221 (1988); S. Dawson, Radiative corrections to Higgs boson production, Nucl. Phys. B359, 283 (1991); A. Djouadi, M. Spira, and P. Zerwas, Production of Higgs bosons in proton colliders. QCD corrections, Phys. Lett. B 264, 440 (1991); M. Spira, A. Djouadi, D. Graudenz, and P. Zerwas, Higgs boson production at the LHC, Nucl. Phys. B453, 17 (1995).

[6] M. Aaboud et al. (ATLAS Collaboration), Observation of $H \rightarrow b \bar{b}$ decays and $V H$ production with the ATLAS detector, Phys. Lett. B 786, 59 (2018); A. M. Sirunyan et al. (CMS Collaboration), Combined measurements of Higgs boson couplings in proton-proton collisions at $\sqrt{s}=13 \mathrm{TeV}$, Eur. Phys. J. C 79, 421 (2019).

[7] M. Aaboud et al. (ATLAS Collaboration), Search for the Decay of the Higgs Boson to Charm Quarks with the ATLAS Experiment, Phys. Rev. Lett. 120, 211802 (2018). 
[8] A. M. Sirunyan et al. (CMS Collaboration), Search for the associated production of the Higgs boson and a vector boson in proton-proton collisions at $\sqrt{s}=13 \mathrm{TeV}$ via Higgs boson decays to $\tau$ leptons, J. High Energy Phys. 06 (2019) 093; M. Aaboud et al. (ATLAS Collaboration), Cross-section measurements of the Higgs boson decaying into a pair of $\tau$-leptons in proton-proton collisions at $\sqrt{s}=13 \mathrm{TeV}$ with the ATLAS detector, Phys. Rev. D 99, 072001 (2019); A. M. Sirunyan et al. (CMS Collaboration), Observation of the Higgs boson decay to a pair of $\tau$ leptons with the CMS detector, Phys. Lett. B 779, 283 (2018).

[9] A. M. Sirunyan et al. (CMS Collaboration), Search for the Higgs Boson Decaying to Two Muons in Proton-Proton Collisions at $\sqrt{s}=13 \mathrm{TeV}$, Phys. Rev. Lett. 122, 021801 (2019); M. Aaboud et al. (ATLAS Collaboration), Search for the Dimuon Decay of the Higgs Boson in $p p$ Collisions at $\sqrt{s}=13 \mathrm{TeV}$ with the ATLAS Detector, Phys. Rev. Lett. 119, 051802 (2017).

[10] V. Khachatryan et al. (CMS Collaboration), Search for a standard model-like Higgs boson in the $\mu^{+} \mu^{-}$and $e^{+} e^{-}$ decay channels at the LHC, Phys. Lett. B 744, 184 (2015); G. Aad et al. (ATLAS Collaboration), Search for the Higgs boson decays $H \rightarrow e e$ and $H \rightarrow e \mu$ in $p p$ collisions at $\sqrt{s}=$ $13 \mathrm{TeV}$ with the ATLAS detector, Phys. Lett. B 801, 135148 (2020).

[11] D. Choudhury and D. P. Roy, Signatures of an invisibly decaying Higgs particle at LHC, Phys. Lett. B 322, 368 (1994).

[12] D. Lee, W. S. Cho, and H. D. Kim, Boosting the searches of gluon pair initiated processes using deep learning of isr jets, Proc. Sci., ICHEP2018 (2019) 796.

[13] G. Kasieczka, N. Kiefer, T. Plehn, and J. M. Thompson, Quark-gluon tagging: Machine learning vs detector, SciPost Phys. 6, 069 (2019).

[14] Y. Chikashige, R. N. Mohapatra, and R. Peccei, Are there real goldstone bosons associated with broken lepton number?, Phys. Lett. B 98, 265 (1981); G. Gelmini and M. Roncadelli, Left-handed neutrino mass scale and spontaneously broken lepton number, Phys. Lett. B 99, 411 (1981).

[15] R. E. Shrock and M. Suzuki, Invisible Decays of Higgs Bosons, Phys. Lett. 110B, 250 (1982); L.-F. Li, Y. Liu, and L. Wolfenstein, Hidden Higgs Particles, Phys. Lett. 159B, 45 (1985); G. Jungman and M. Luty, Massive neutrinos and the weak scale singlet Majoron, Nucl. Phys. B361, 24 (1991); A. S. Joshipura and S. D. Rindani, Majoron Models and the Higgs Search, Phys. Rev. Lett. 69, 3269 (1992); A. S. Joshipura and J. Valle, Invisible Higgs decays and neutrino physics, Nucl. Phys. B397, 105 (1993).

[16] K. Griest and H. E. Haber, Invisible decays of Higgs bosons in supersymmetric models, Phys. Rev. D 37, 719 (1988); J. F. Gunion and H. E. Haber, Higgs bosons in supersymmetric models. 3. decays into neutralinos and charginos, Nucl. Phys. B307, 445 (1988); B402, 569(E) (1993); J. Romao, F. de Campos, and J. Valle, New Higgs signatures in supersymmetry with spontaneous broken R parity, Phys. Lett. B 292, 329 (1992); F. de Campos, M. Garcia-Jareno, A. S. Joshipura, J. Rosiek, and J. Valle, Novel scalar boson decays in SUSY with broken r parity, Nucl. Phys. B451, 3 (1995); A. Djouadi, P. Janot, J. Kalinowski, and P. Zerwas,
SUSY decays of Higgs particles, Phys. Lett. B 376, 220 (1996); A. Djouadi and M. Drees, Higgs boson decays into light gravitinos, Phys. Lett. B 407, 243 (1997); G. Belanger, F. Boudjema, A. Cottrant, R. M. Godbole, and A. Semenov, The MSSM invisible Higgs in the light of dark matter and g-2, Phys. Lett. B 519, 93 (2001); M. Hirsch, J. Romao, J. Valle, and A. Villanova del Moral, Invisible Higgs boson decays in spontaneously broken R-parity, Phys. Rev. D 70, 073012 (2004); I. Antoniadis, M. Tuckmantel, and F. Zwirner, Phenomenology of a leptonic goldstino and invisible Higgs boson decays, Nucl. Phys. B707, 215 (2005); S. Chang, P. J. Fox, and N. Weiner, Naturalness and Higgs decays in the MSSM with a singlet, J. High Energy Phys. 08 (2006) 068; P. Draper, T. Liu, C. E. Wagner, L.-T. Wang, and H. Zhang, Dark Light Higgs, Phys. Rev. Lett. 106, 121805 (2011); J.-J. Cao, Z. Heng, J. M. Yang, and J. Zhu, Higgs decay to dark matter in low energy SUSY: is it detectable at the LHC?, J. High Energy Phys. 06 (2012) 145; A. Butter, T. Plehn, M. Rauch, D. Zerwas, S. Henrot-Versill, and R. Lafaye, Invisible Higgs decays to hooperons in the NMSSM, Phys. Rev. D 93, 015011 (2016); R. K. Barman, G. Belanger, B. Bhattacherjee, R. Godbole, G. Mendiratta, and D. Sengupta, Invisible decay of the Higgs boson in the context of a thermal and nonthermal relic in MSSM, Phys. Rev. D 95, 095018 (2017); K. Wang and J. Zhu, Funnel annihilations of light dark matter and the invisible decay of the Higgs boson, Phys. Rev. D 101, 095028 (2020); R. K. Barman, G. Blanger, B. Bhattacherjee, R. Godbole, D. Sengupta, and X. Tata, urrent bounds and future prospects of light neutralino dark matter in NMSSM, arXiv:2006.07854.

[17] A. Pilaftsis, Radiatively induced neutrino masses and large Higgs-neutrino couplings in the Standard Model with Majorana fields, Z. Phys. C 55, 275 (1992).

[18] N. Arkani-Hamed, S. Dimopoulos, and G. Dvali, The Hierarchy problem and new dimensions at a millimeter, Phys. Lett. B 429, 263 (1998).

[19] K. R. Dienes, E. Dudas, and T. Gherghetta, Neutrino oscillations without neutrino masses or heavy mass scales: A Higher dimensional seesaw mechanism, Nucl. Phys. B557, 25 (1999); N. Arkani-Hamed, S. Dimopoulos, G. Dvali, and J. March-Russell, Neutrino masses from large extra dimensions, Phys. Rev. D 65, 024032 (2001); G. F. Giudice, R. Rattazzi, and J. D. Wells, Graviscalars from higher dimensional metrics and curvature Higgs mixing, Nucl. Phys. B595, 250 (2001); M. Battaglia, D. Dominici, J. Gunion, and J. Wells, The Invisible Higgs decay width in the add model at the LHC, arXiv:hep-ph/0402062; A. Datta, K. Huitu, J. Laamanen, and B. Mukhopadhyaya, Invisible Higgs in theories of large extra dimensions, Phys. Rev. D 70, 075003 (2004); D. Dominici and J. F. Gunion, Invisible Higgs decays from Higgs graviscalar mixing, Phys. Rev. D 80, 115006 (2009).

[20] D. Fargion, Y. Golubkov, M. Khlopov, R. Konoplich, and R. Mignani, Possible effects of the existence of the fourth generation neutrino, JETP Lett. 69, 434 (1999); V. A. Khoze, Comment on an invisible Higgs boson and 50 GeV neutrino, arXiv:hep-ph/0105069; K. Belotsky, D. Fargion, M. Khlopov, R. Konoplich, and K. Shibaev, 
Invisible Higgs boson decay into massive neutrinos of fourth generation, Phys. Rev. D 68, 054027 (2003).

[21] K. Belotsky, V. Khoze, A. Martin, and M. Ryskin, Can an invisible Higgs boson be seen via diffraction at the LHC?, Eur. Phys. J. C 36, 503 (2004).

[22] J.-M. Frere, A. Rozanov, and M. Vysotsky, Mass and decays of Brout-Englert-Higgs scalar with extra generations, Phys. At. Nucl. 69, 355 (2006); G. D. Kribs, T. Plehn, M. Spannowsky, and T. M. Tait, Four generations and Higgs physics, Phys. Rev. D 76, 075016 (2007); O. Eberhardt, G. Herbert, H. Lacker, A. Lenz, A. Menzel, U. Nierste, and M. Wiebusch, Joint analysis of Higgs decays and electroweak precision observables in the Standard Model with a sequential fourth generation, Phys. Rev. D 86, 013011 (2012).

[23] V. Silveira and A. Zee, Scalar Phantoms, Phys. Lett. B 161, 136 (1985); N. Krasnikov, Invisible scalars visible in Higgs decay, Phys. Lett. B 291, 89 (1992); J. McDonald, Gauge singlet scalars as cold dark matter, Phys. Rev. D 50, 3637 (1994); T. Binoth and J. van der Bij, Influence of strongly coupled, hidden scalars on Higgs signals, Z. Phys. C 75, 17 (1997); A. Datta and A. Raychaudhuri, Next-to-minimal Higgs: Mass bounds and search prospects, Phys. Rev. D 57, 2940 (1998); C. Burgess, M. Pospelov, and T. ter Veldhuis, The Minimal model of nonbaryonic dark matter: A Singlet scalar, Nucl. Phys. B619, 709 (2001); M. Bento, O. Bertolami, and R. Rosenfeld, Cosmological constraints on an invisibly decaying Higgs boson, Phys. Lett. B 518, 276 (2001).

[24] H. Davoudias1, R. Kitano, T. Li, and H. Murayama, The New minimal standard model, Phys. Lett. B 609, 117 (2005); R. M. Schabinger and J. D. Wells, A Minimal spontaneously broken hidden sector and its impact on Higgs boson physics at the large hadron collider, Phys. Rev. D 72, 093007 (2005); R. Barbieri, T. Gregoire, and L. J. Hall, Mirror world at the large hadron collider, arXiv:hep-ph/ 0509242; B. Patt and F. Wilczek, Higgs-field portal into hidden sectors, arXiv:hep-ph/0605188; D. Cerdeno, A. Dedes, and T. Underwood, The minimal phantom sector of the standard model: Higgs phenomenology and dirac leptogenesis, J. High Energy Phys. 09 (2006) 067; D. O'Connell, M. J. Ramsey-Musolf, and M. B. Wise, Minimal extension of the standard model scalar sector, Phys. Rev. D 75, 037701 (2007); Y. G. Kim and K. Y. Lee, The Minimal model of fermionic dark matter, Phys. Rev. D 75, 115012 (2007); O. Bahat-Treidel, Y. Grossman, and Y. Rozen, Hiding the Higgs at the LHC, J. High Energy Phys. 05 (2007) 022; V. Barger, P. Langacker, M. McCaskey, M. J. Ramsey-Musolf, and G. Shaughnessy, LHC phenomenology of an extended standard model with a real scalar singlet, Phys. Rev. D 77, 035005 (2008); O. Bertolami and R. Rosenfeld, The Higgs portal and an unified model for dark energy and dark matter, Int. J. Mod. Phys. A 23, 4817 (2008); Y. G. Kim, K. Y. Lee, and S. Shin, Singlet fermionic dark matter, J. High Energy Phys. 05 (2008) 100; V. Barger, P. Langacker, M. McCaskey, M. Ramsey-Musolf, and G. Shaughnessy, Complex singlet extension of the standard model, Phys. Rev. D 79, 015018 (2009); M. Gonderinger, Y. Li, H. Patel, and M. J. Ramsey-Musolf, Vacuum stability, perturbativity, and scalar singlet dark matter, J. High Energy Phys. 01 (2010) 053; K. Cheung and T.-C.
Yuan, Implication on Higgs invisible width in light of the new CDMS result, Phys. Lett. B 685, 182 (2010); X.-G. He, T. Li, X.-Q. Li, J. Tandean, and H.-C. Tsai, The Simplest Dark-Matter Model, CDMS II Results, and Higgs Detection at LHC, Phys. Lett. B 688, 332 (2010); S. Andreas, C. Arina, T. Hambye, F.-S. Ling, and M. H. Tytgat, A light scalar WIMP through the Higgs portal and CoGeNT, Phys. Rev. D 82, 043522 (2010); S. Kanemura, S. Matsumoto, T. Nabeshima, and N. Okada, Can WIMP dark matter overcome the nightmare ccenario?, Phys. Rev. D 82, 055026 (2010); C. Englert, T. Plehn, D. Zerwas, and P. M. Zerwas, Exploring the Higgs portal, Phys. Lett. B 703, 298 (2011); M. Raidal and A. Strumia, Hints for a non-standard Higgs boson from the LHC, Phys. Rev. D 84, 077701 (2011); X.G. He and J. Tandean, Hidden Higgs boson at the LHC and light dark matter searches, Phys. Rev. D 84, 075018 (2011); M. Pospelov and A. Ritz, Higgs decays to dark matter: Beyond the minimal model, Phys. Rev. D 84, 113001 (2011); O. Lebedev, H. M. Lee, and Y. Mambrini, Vector Higgs-portal dark matter and the invisible Higgs, Phys. Lett. B 707, 570 (2012); S. Baek, P. Ko, and W.-I. Park, Search for the Higgs portal to a singlet fermionic dark matter at the LHC, J. High Energy Phys. 02 (2012) 047; A. Drozd, B. Grzadkowski, and J. Wudka, Multi-scalar-singlet extension of the standard model - the case for dark matter and an invisible Higgs boson, J. High Energy Phys. 04 (2012) 006; 11 (2014) 130; A. Djouadi, O. Lebedev, Y. Mambrini, and J. Quevillon, Implications of LHC searches for Higgs-portal dark matter, Phys. Lett. B 709, 65 (2012); B. Batell, S. Gori, and L.-T. Wang, Exploring the Higgs portal with 10/fb at the LHC, J. High Energy Phys. 06 (2012) 172; X.-G. He, B. Ren, and J. Tandean, Hints of standard model Higgs boson at the LHC and light dark matter searches, Phys. Rev. D 85, 093019 (2012); J. F. Kamenik and C. Smith, Could a light Higgs boson illuminate the dark sector?, Phys. Rev. D 85, 093017 (2012).

[25] A. Djouadi, A. Falkowski, Y. Mambrini, and J. Quevillon, Direct Detection of Higgs-Portal Dark Matter at the LHC, Eur. Phys. J. C 73, 2455 (2013).

[26] J. M. Cline, K. Kainulainen, P. Scott, and C. Weniger, Update on scalar singlet dark matter, Phys. Rev. D 88, 055025 (2013); 92, 039906(E) (2015); A. De Simone, G. F. Giudice, and A. Strumia, Benchmarks for dark matter searches at the LHC, J. High Energy Phys. 06 (2014) 081; S. Baek, P. Ko, and W.-I. Park, Invisible Higgs decay width vs. dark matter direct detection cross section in Higgs portal dark matter models, Phys. Rev. D 90, 055014 (2014); L. Feng, S. Profumo, and L. Ubaldi, Closing in on singlet scalar dark matter: LUX, invisible Higgs decays and gamma-ray lines, J. High Energy Phys. 03 (2015) 045; A. Beniwal, F. Rajec, C. Savage, P. Scott, C. Weniger, M. White, and A. G. Williams, Combined analysis of effective Higgs portal dark matter models, Phys. Rev. D 93, 115016 (2016); H. Han, J. M. Yang, Y. Zhang, and S. Zheng, Collider signatures of Higgs-portal scalar dark matter, Phys. Lett. B 756, 109 (2016); G. Dupuis, Collider constraints and prospects of a scalar singlet extension to Higgs portal dark matter, J. High Energy Phys. 07 (2016) 008; G. Arcadi, A. Djouadi, and M. Raidal, Dark Matter through the Higgs portal, Phys. Rep. 842, 1 (2020). 
[27] S. Frederiksen, N. Johnson, G. L. Kane, and J. Reid, Detecting invisible Higgs bosons at the CERN Large Hadron Collider, Phys. Rev. D 50, R4244 (1994).

[28] H. Davoudiasl, T. Han, and H. E. Logan, Discovering an invisibly decaying Higgs at hadron colliders, Phys. Rev. D 71, 115007 (2005); S. P. Martin and J. D. Wells, Motivation and detectability of an invisibly decaying Higgs boson at the Fermilab Tevatron, Phys. Rev. D 60, 035006 (1999); R. M. Godbole, M. Guchait, K. Mazumdar, S. Moretti, and D. P. Roy, Search for 'invisible' Higgs signals at LHC via associated production with gauge bosons, Phys. Lett. B 571, 184 (2003).

[29] C. Englert, J. Jaeckel, E. Re, and M. Spannowsky, Evasive Higgs boson maneuvers at the LHC, Phys. Rev. D 85, 035008 (2012).

[30] Y. Bai, P. Draper, and J. Shelton, Measuring the invisible Higgs width at the 7 and $8 \mathrm{TeV}$ LHC, J. High Energy Phys. 07 (2012) 192.

[31] D. Ghosh, R. Godbole, M. Guchait, K. Mohan, and D. Sengupta, Looking for an invisible Higgs signal at the LHC, Phys. Lett. B 725, 344 (2013);

[32] L. M. Carpenter, A. Nelson, C. Shimmin, T. M. Tait, and D. Whiteson, Collider searches for dark matter in events with a $Z$ boson and missing energy, Phys. Rev. D 87, 074005 (2013); D. Goncalves, F. Krauss, S. Kuttimalai, and P. Maierhfer, Boosting invisible searches via $\mathrm{ZH}$ : From the Higgs boson to dark matter simplified models, Phys. Rev. D 94, 053014 (2016); D. Goncalves and J. Nakamura, Boosting the $H \rightarrow$ invisibles searches with $Z$ boson polarization, Phys. Rev. D 99, 055021 (2019).

[33] G. Aad et al. (ATLAS Collaboration), Search for dark matter in events with a hadronically decaying $W$ or $Z$ boson and missing transverse momentum in $p p$ collisions at $\sqrt{s}=$ $8 \mathrm{TeV}$ with the ATLAS detector, Phys. Rev. Lett. 112, 041802 (2014); 112, 201802(E) (2014); Search for dark matter in events with a $Z$ boson and missing transverse momentum in pp collisions at $\sqrt{s}=8 \mathrm{TeV}$ with the ATLAS detector, Phys. Rev. D 90, 012004 (2014); Search for invisible decays of the Higgs boson produced in association with a hadronically decaying vector boson in $p p$ collisions at $\sqrt{s}=8 \mathrm{TeV}$ with the ATLAS detector, Eur. Phys. J. C 75, 337 (2015); Search for invisible Higgs bosons in pp $\rightarrow$ $\mathrm{ZH} \rightarrow 2 \ell+E_{\mathrm{T}}^{\text {miss }}$ channels at $\sqrt{s}=13 \mathrm{TeV}$ (2016), https:// cds.cern.ch/record/2138371; M. Aaboud et al. (ATLAS Collaboration), Search for an invisibly decaying Higgs boson or dark matter candidates produced in association with a $Z$ boson in $p p$ collisions at $\sqrt{s}=13 \mathrm{TeV}$ with the ATLAS detector, Phys. Lett. B 776, 318 (2018); , Search for dark matter in events with a hadronically decaying vector boson and missing transverse momentum in $p p$ collisions at $\sqrt{s}=13 \mathrm{TeV}$ with the ATLAS detector, J. High Energy Phys. 10 (2018) 180.

[34] (e.g. see Table. 7 and Fig. 5) V. Khachatryan et al. (CMS Collaboration), Searches for invisible decays of the Higgs boson in pp collisions at $\sqrt{s}=7,8$, and $13 \mathrm{TeV}$, J. High Energy Phys. 02 (2017) 135.

[35] (e.g. see Table. 4) A. M. Sirunyan et al. (CMS Collaboration), Search for new physics in final states with an energetic jet or a hadronically decaying $W$ or $Z$ boson and transverse momentum imbalance at $\sqrt{s}=13 \mathrm{TeV}$, Phys. Rev. D 97, 092005 (2018).

[36] S. Chatrchyan et al. (CMS Collaboration), Search for invisible decays of Higgs bosons in the vector boson fusion and associated $\mathrm{ZH}$ production modes, Eur. Phys. J. C 74, 2980 (2014).

[37] ATLAS Collaboration, Combination of searches for invisible Higgs boson decays with the ATLAS experiment (2018).

[38] M. Aaboud et al. (ATLAS Collaboration), Combination of searches for invisible Higgs boson decays with the ATLAS experiment, Phys. Rev. Lett. 122, 231801 (2019).

[39] J. F. Gunion, Detecting an invisibly decaying Higgs boson at a hadron supercollider, Phys. Rev. Lett. 72, 199 (1994).

[40] B. P. Kersevan, M. Malawski, and E. Richter-Was, Prospects for observing an invisibly decaying Higgs boson in the $t$ anti-t $\mathrm{H}$ production at the LHC, Eur. Phys. J. C 29, 541 (2003); M. Malawski, Master thesis, Jagiellonian University, 2004; N. Zhou, Z. Khechadoorian, D. Whiteson, and T. M. Tait, Bounds on Invisible Higgs boson Decays from $t \bar{t} H$ Production, Phys. Rev. Lett. 113, 151801 (2014); 114, 229901(E) (2015); N. Craig, F. D'Eramo, P. Draper, S. Thomas, and H. Zhang, The Hunt for the Rest of the Higgs Bosons, J. High Energy Phys. 06 (2015) 137; M. R. Buckley and D. Goncalves, Constraining the strength and $C P$ structure of dark production at the LHC: The associated top-pair channel, Phys. Rev. D 93, 034003 (2016).

[41] CMS Collaboration, First constraints on invisible Higgs boson decays using $\mathrm{t} \overline{\mathrm{t}} \mathrm{H}$ production at $\sqrt{s}=13 \mathrm{TeV}$ (2019).

[42] P. J. Fox, R. Harnik, J. Kopp, and Y. Tsai, Missing energy signatures of dark matter at the LHC, Phys. Rev. D 85, 056011 (2012).

[43] D. Barducci, A. Bharucha, N. Desai, M. Frigerio, B. Fuks, A. Goudelis, S. Kulkarni, G. Polesello, and D. Sengupta, Monojet searches for momentum-dependent dark matter interactions, J. High Energy Phys. 01 (2017) 078.

[44] S. Chatrchyan et al. (CMS Collaboration), Search for new physics with a mono-jet and missing transverse energy in $p p$ collisions at $\sqrt{s}=7 \mathrm{TeV}$, Phys. Rev. Lett. 107, 201804 (2011); G. Aad et al. (ATLAS Collaboration), Search for new phenomena with the monojet and missing transverse momentum signature using the ATLAS detector in $\sqrt{s}=7$ $\mathrm{TeV}$ proton-proton collisions, Phys. Lett. B 705, 294 (2011); T. Aaltonen et al. (CDF Collaboration), A Search for dark matter in events with one jet and missing transverse energy in $p \bar{p}$ collisions at $\sqrt{s}=1.96 \mathrm{TeV}$, Phys. Rev. Lett. 108, 211804 (2012); S. Chatrchyan et al. (CMS Collaboration), Search for dark matter and large extra dimensions in monojet events in $p p$ collisions at $\sqrt{s}=7 \mathrm{TeV}$, J. High Energy Phys. 09 (2012) 094; Search for new phenomena in monojet plus missing transverse momentum final states using $10 \mathrm{fb}^{-1}$ of pp collisions at $\sqrt{s}=8 \mathrm{TeV}$ with the ATLAS detector at the LHC (2012), https://cds.cern.ch/record/ 1493486; G. Aad et al. (ATLAS Collaboration), Search for dark matter candidates and large extra dimensions in events with a jet and missing transverse momentum with the ATLAS detector, J. High Energy Phys. 04 (2013) 075; V. Khachatryan et al. (CMS Collaboration), Search for dark matter, extra dimensions, and unparticles in monojet events in proton-proton collisions at $\sqrt{s}=8 \mathrm{TeV}$, Eur. Phys. J. C 
75, 235 (2015); G. Aad et al. (ATLAS Collaboration), Search for new phenomena in final states with an energetic jet and large missing transverse momentum in pp collisions at $\sqrt{s}=8 \mathrm{TeV}$ with the ATLAS detector, Eur. Phys. J. C 75, 299 (2015); 75, 408(E) (2015); M. Aaboud et al. (ATLAS Collaboration), Search for new phenomena in final states with an energetic jet and large missing transverse momentum in $p p$ collisions at $\sqrt{s}=13 \mathrm{TeV}$ using the ATLAS detector, Phys. Rev. D 94, 032005 (2016); A. M. Sirunyan et al. (CMS Collaboration), Search for dark matter produced with an energetic jet or a hadronically decaying $\mathrm{W}$ or $\mathrm{Z}$ boson at $\sqrt{s}=13$ TeV, J. High Energy Phys. 07 (2017) 014.

[45] O. J. P. Eboli and D. Zeppenfeld, Observing an invisible Higgs boson, Phys. Lett. B 495, 147 (2000).

[46] C. Bernaciak, T. Plehn, P. Schichtel, and J. Tattersall, Spying an invisible Higgs boson, Phys. Rev. D 91, 035024 (2015); D. Goncalves, T. Plehn, and J. M. Thompson, Weak boson fusion at $100 \mathrm{TeV}$, Phys. Rev. D 95, 095011 (2017); A. Biektter, F. Keilbach, R. Moutafis, T. Plehn, and J. Thompson, Tagging jets in invisible Higgs searches, SciPost Phys. 4, 035 (2018); J. Heisig, M. Krmer, E. Madge, and A. Mck, Probing Higgs-portal dark matter with vector-boson fusion, J. High Energy Phys. 03 (2020) 183.

[47] B. Di Girolamo and L. Neukermans, Observing an invisibly decaying Higgs boson in ATLAS via vector boson fusion (2002), http://cds.cern.ch/record/685420; G. Aad et al. (ATLAS Collaboration), Search for invisible decays of a Higgs boson using vector-boson fusion in $p p$ collisions at $\sqrt{s}=8 \mathrm{TeV}$ with the ATLAS detector, J. High Energy Phys. 01 (2016) 172; Constraints on new phenomena via Higgs boson couplings and invisible decays with the ATLAS detector, 11 (2015) 206; Search for invisible decays of a Higgs boson produced via vector boson fusion at $\sqrt{s}=$ $13 \mathrm{TeV}$ (2016), https://cds.cern.ch/record/2142460; M. Aaboud et al. (ATLAS Collaboration), Search for invisible Higgs boson decays in vector boson fusion at $\sqrt{s}=13 \mathrm{TeV}$ with the ATLAS detector, Phys. Lett. B 793, 499 (2019); Phys. Lett. BSearch for invisible Higgs boson decays with vector boson fusion signatures with the ATLAS detector using an integrated luminosity of $139 \mathrm{fb}^{-1}$ (2020), https:// cds.cern.ch/record/2715447.

[48] A. M. Sirunyan et al. (CMS Collaboration), Search for invisible decays of a Higgs boson produced through vector boson fusion in proton-proton collisions at $\sqrt{s}=13 \mathrm{TeV}$, Phys. Lett. B 793, 520 (2019).

[49] I. Low, P. Schwaller, G. Shaughnessy, and C. E. Wagner, The dark side of the Higgs boson, Phys. Rev. D 85, 015009 (2012).

[50] C. Kim, S. C. Park, K. Wang, and G. Zhu, Invisible Higgs boson decay with $B \rightarrow K \nu \bar{\nu}$, Phys. Rev. D 81, 054004 (2010).

[51] S. Banerjee, B. Batell, and M. Spannowsky, Invisible decays in Higgs boson pair production, Phys. Rev. D 95, 035009 (2017); C. Englert and J. Jaeckel, Probing the symmetric Higgs portal with di-Higgs boson production, Phys. Rev. D 100, 095017 (2019).

[52] M. Endo and Y. Takaesu, Heavy WIMP through Higgs portal at the LHC, Phys. Lett. B 743, 228 (2015); N. Craig, H. K. Lou, M. McCullough, and A. Thalapillil, The Higgs portal above threshold, J. High Energy Phys. 02 (2016) 127;
M. Ruhdorfer, E. Salvioni, and A. Weiler, A global view of the off-shell Higgs portal, SciPost Phys. 8, 027 (2020).

[53] O. J. Eboli, M. Gonzalez-Garcia, A. Lopez-Fernandez, S. Novaes, and J. Valle, Searching for an invisibly decaying Higgs boson in $e^{+} e^{-}$, e gamma and gamma gamma collisions, Nucl. Phys. B421, 65 (1994); arXiv:hepex/0107032; J. Abdallah et al. (DELPHI Collaboration), Searches for invisibly decaying Higgs bosons with the DELPHI detector at LEP, Eur. Phys. J. C 32, 475 (2004); G. Aarons et al. (ILC Collaboration), International linear collider reference design report Volume 2: physics at the ILC, arXiv:0709.1893; S. Kanemura, S. Matsumoto, T. Nabeshima, and H. Taniguchi, Testing Higgs portal dark matter via $Z$ fusion at a linear collider, Phys. Lett. B 701, 591 (2011); S. Kanemura, S. Matsumoto, T. Nabeshima, and H. TaniguchiThe international linear collider technical design report Volume 2: physics, arXiv:1306.6352; A. Arbey et al., Physics at the $e^{+} e^{-}$linear collider, Eur. Phys. J. C 75, 371 (2015); Y. Kato, Probing the dark sector via searches for invisible decays of the Higgs boson at the ILC, arXiv:2002.12048; Y.-L. Tang, C. Zhang, and S.-h. Zhu, Invisible Higgs Decay at the LHeC, Phys. Rev. D 94, 011702 (2016).

[54] C. Englert, T. Plehn, M. Rauch, D. Zerwas, and P. M. Zerwas, LHC: Standard Higgs and hidden Higgs, Phys. Lett. B 707, 512 (2012); J. R. Espinosa, M. Muhlleitner, C. Grojean, and M. Trott, Probing for invisible higgs decays with global fits, J. High Energy Phys. 09 (2012) 126; G. Belanger, B. Dumont, U. Ellwanger, J. F. Gunion, and S. Kraml, Status of invisible Higgs decays, Phys. Lett. B 723, 340 (2013); P. Bechtle, O. Brein, S. Heinemeyer, O. Stl, T. Stefaniak, G. Weiglein, and K. E. Williams, HiggsBounds - 4: Improved tests of extended Higgs sectors against exclusion bounds from LEP, the Tevatron and the LHC, Eur. Phys. J. C 74, 2693 (2014); D. Curtin et al., Exotic decays of the $125 \mathrm{GeV}$ Higgs boson, Phys. Rev. D 90, 075004 (2014); T. Corbett, O. J. P. Eboli, D. Goncalves, J. Gonzalez-Fraile, T. Plehn, and M. Rauch, The Higgs Legacy of the LHC Run I, J. High Energy Phys. 08 (2015) 156; P. Athron et al. (GAMBIT Collaboration), Global analyses of Higgs portal singlet dark matter models using GAMBIT, Eur. Phys. J. C 79, 38 (2019); C. Arina, A. Beniwal, C. Degrande, J. Heisig, and A. Scaffidi, Global fit of pseudo-Nambu-Goldstone Dark Matter, J. High Energy Phys. 04 (2020) 015.

[55] J. Alwall, R. Frederix, S. Frixione, V. Hirschi, F. Maltoni, O. Mattelaer, H. S. Shao, T. Stelzer, P. Torrielli, and M. Zaro, The automated computation of tree-level and next-to-leading order differential cross sections, and their matching to parton shower simulations, J. High Energy Phys. 07 (2014) 079.

[56] T. Sjstrand, S. Ask, J. R. Christiansen, R. Corke, N. Desai, P. Ilten, S. Mrenna, S. Prestel, C. O. Rasmussen, and P.Z. Skands, An introduction to PYTHIA 8.2, Comput. Phys. Commun. 191, 159 (2015).

[57] J. de Favereau, C. Delaere, P. Demin, A. Giammanco, V. Lematre, A. Mertens, and M. Selvaggi (DELPHES 3 Collaboration), DELPHES 3, A modular framework for fast simulation of a generic collider experiment, J. High Energy Phys. 02 (2014) 057.

[58] V. Hirschi and O. Mattelaer, Automated event generation for loop-induced processes, J. High Energy Phys. 10 (2015) 146. 
[59] R. Frederix and S. Frixione, Merging meets matching in MC@NLO, J. High Energy Phys. 12 (2012) 061.

[60] M. Cacciari, G. P. Salam, and G. Soyez, FastJet user manual, Eur. Phys. J. C 72, 1896 (2012).

[61] H.-L. Lai, M. Guzzi, J. Huston, Z. Li, P. M. Nadolsky, J. Pumplin, and C.P. Yuan, New parton distributions for collider physicsPhys. Rev. D 82, 074024 (2010).

[62] S. D. Ellis, C. K. Vermilion, and J. R. Walsh, Recombination algorithms and jet substructure: Pruning as a tool for heavy particle searches, Phys. Rev. D 81, 094023 (2010).

[63] J. Thaler and K. Van Tilburg, Identifying boosted objects with $N$-subjettiness, J. High Energy Phys. 03 (2011) 015.

[64] A. J. Larkoski, I. Moult, and B. Nachman, Jet substructure at the large hadron collider: A review of recent advances in theory and machine learning, Phys. Rep. 841, 1 (2020).

[65] J. Gallicchio and M. D. Schwartz, Quark and Gluon Tagging at the LHC, Phys. Rev. Lett. 107, 172001 (2011).

[66] J. Gallicchio, J. Huth, M. Kagan, M. D. Schwartz, K. Black, and B. Tweedie, Multivariate discrimination and the Higgs +W/Z search, J. High Energy Phys. 04 (2011) 069.

[67] S. Catani, G. Turnock, and B. R. Webber, Jet broadening measures in $e^{+} e^{-}$annihilation, Phys. Lett. B 295, 269 (1992).

[68] B. Bhattacherjee, S. Mukhopadhyay, M. M. Nojiri, Y. Sakaki, and B. R. Webber, Quark-gluon discrimination in the search for gluino pair production at the LHC, J. High Energy Phys. 01 (2017) 044.
[69] A. J. Larkoski, G. P. Salam, and J. Thaler, Energy correlation functions for jet substructure, J. High Energy Phys. 06 (2013) 108.

[70] P. T. Komiske, E. M. Metodiev, and M. D. Schwartz, Deep learning in color: Towards automated quark/gluon jet discrimination, J. High Energy Phys. 01 (2017) 110.

[71] J. Cogan, M. Kagan, E. Strauss, and A. Schwarztman, Jet-Images: Computer vision inspired techniques for jet tagging, J. High Energy Phys. 02 (2015) 118.

[72] F. Chollet et al., Keras, https://keras.io (2015).

[73] ATLAS, CMS, LHC Higgs Combination Group, Procedure for the LHC Higgs boson search combination in summer 2011 (2011).

[74] D. de Florian et al. (LHC Higgs Cross Section Working Group), Handbook of LHC Higgs Cross Sections: 4. Deciphering the Nature of the Higgs Sector (CERN, Geneva, 2017), Vol. 2.

[75] S. Ioffe and C. Szegedy, Batch normalization: Accelerating deep network training by reducing internal covariate shift, arXiv:1502.03167.

[76] D. P. Kingma and J. Ba, Adam: A method for stochastic optimization, arXiv:1412.6980.

[77] K. He, X. Zhang, S. Ren, and J. Sun, Delving deep into rectifiers: Surpassing human-level performance on ImageNet classification, arXiv:1502.01852.

[78] P. Artoisenet et al., A framework for Higgs characterisation, J. High Energy Phys. 11 (2013) 043. 